\title{
Análisis de la red vial de la provincia de Corrientes por medio de la Teoría de Grafos
}

\author{
Prof. Juan Ariel Insaurralde \\ Becario de CONICET - Departamento de Geografía y Turismo (UNS) \\ insaurraldejuan@conicet.gov.ar
}

Prof. Osvaldo Daniel Cardozo

Prof. Adjunto. Departamento de Geografía - LabTIG - GEMSIT

Facultad de Humanidades - UNNE

odcardozo@hum.unne.ar

\begin{abstract}
Resumen
El objetivo del presente trabajo es analizar por medio de la Teoría de Grafos la red vial de la Provincia de Corrientes, para determinar desigualdades en la accesibilidad, conectividad y centralidad de la misma. Según esta Teoría el espacio geográfico se reduce a un grafo, es decir, un dibujo compuesto por arcos y nodos que representan a elementos de la realidad, como rutas y las intersecciones de las mismas o ciudades respectivamente. Generado el grafo a partir de las rutas pavimentadas, se procede a la construcción de las matrices de datos (conectividad y accesibilidad), al cálculo de índices con diferentes niveles de complejidad, y la elaboración de una cartografía que expresa las relaciones topológicas. Pese a su carácter preferentemente descriptivo, es posible evidenciar algunos desequilibrios en la estructura espacial de la red vial.
\end{abstract}

Palabras clave: Provincia de Corrientes; Grafos; Accesibilidad; Centralidad; Conectividad

\section{Analysis of road network in the province of Corrientes through the Graphstheory}

\section{Summary}

The objective of the present work is to analyze through the Graph Theory the road network in the Provincia de Corrientes, to determine same disparities in accessibility, connectivity and centrality. According to this Theory the geographic space is reduced to a graph, that is to say, a drawing composed of arcs and nodes/links that represent elements of the reality, as the routes and the intersections of the same or cities respectively. Graph generated from paved roads, we proceed to the construction of the data matrix (connectivity and accessibility), the calculation of indices with different levels from complexity, and the elaboration of a mapping that expresses the topological relationships. Although mainly descriptive nature, it is possible to highlight some imbalances in the spatial structure of the road network.

Key words: Provincia de Corrientes; Graph; Accessibility; Centrality; Connectivity. 


\section{SUMARIO}

1. Introducción
1.1. Objetivos
1.2. Área de estudio

2. Marco conceptual-metodológico

2.1. Antecedentes del tema

2.2. Fuentes de Información y Criterios de Elaboración

2.3. Medidas de Conectividad Topológica

2.4. Medidas de Accesibilidad Topológica

2.5. Medidas de Centralidad Topológica

\section{Resultados}

3.1. Análisis de la conectividad, accesibilidad y centralidad.

\section{Conclusiones \\ 5. Bibliografía \\ 6. Anexos}

\section{INTRODUCCIÓN}

La configuración de los espacios geográficos puede explicarse de muchas formas, pero en todas ellas, es indudable el importante rol que desempañan las redes de transporte -e infraestructuras anexas-que se emplazan sobre el mismo. Esta mutua relación es aceptada con un amplio consenso por la comunidad científica, pero cuando se intenta abordar dicho análisis las alternativas de enfoque son diversas, aunque ello -al menos desde nuestra perspectiva- no significa necesariamente que sean incompatibles. Una de ellas es la Teoría de Grafos, que básicamente, propone reducir la realidad a elementos geométricos para explorar sus propiedades topológicas (vecindad, conectividad, etc.).

El antecedente más relevante de la aplicación de este teoríaconstituye el trabajo del matemático sueco Leonhard Euler en 1736, a raíz delproblema denominado "Ios puentes de Königsberg" y continuados luego por Kirchohff con una aplicación a circuitos eléctricos. Sin embargo,recién con la resolución del problema "los cuatro colores"logró consolidarse como una rama de la Topología en el campo de las Matemáticas e Investigación Operativa. En la actualidad, su difusión y creciente interés en campos tan diversos como la Informática, Electrónica, Matemática, Economía, Geografía, Ingeniería, Urbanismo, Sociología, Psicología, o Administración, obedece a que proporciona algoritmos eficientes para resolver problemas en diferentes campos de las ciencias.

En este sentido, un claro reflejo del advenimiento de la revolución cuantitativa en las Ciencias Humanas y con ello, una mayor difusión de la modelización matemática para la resolución de problemáticas de índole espacial, fue la introducción de la Teoría de Grafos en la Geografía con los pioneros trabajos de William L. Garrison 
(1960) y J. Kansky (1963), empleada para el estudio de las redes de comunicaciones y transporte en Estados Unidos.

Independientemente de trabajar con elementos intangibles (información, energía, relaciones) o tangibles (red hidrográfica, carreteras), y de representarlo en forma gráfica o matricial, es necesario reducir la realidad a un grafo, es decir, un dibujo cuyos componentes esenciales son los arcos para elementos lineales y los nodos para los puntuales. Otros datos a tener en cuenta pueden ser la cantidad o volumen de la relación, la dirección de la misma, y una ponderación los nodos a partir de algún tipo de jerarquía.

En términos básicos y pese a su visión abstractaesta teoría es fácilmente comparable con elementos concretos de la realidad, de hecho, sus componentes fundamentales -arcos y nodos- son factibles de ser relacionarlos con hechos geográficos, tales como vías de comunicación, la hidrografía, las paradas de colectivos, las ciudades, terminales informáticas, etc. Sin embargo, es justo reconocer que esta teoría permite básicamente un análisis descriptivo y explicativo aunque esto último con ciertas limitaciones- de la estructura de una red, es decir que permite su estudio de un punto de vista morfométrico.

El modo de proceder para la aplicación de la Teoría de Grafos, es mediante el empleo de diferentes índices y matrices que permitan conocer la estructura de la red, para determinar el grado de conexión entre los nodos o la evolución de tal conexión con el tiempo(Seguí Pons y Petrus Bey, 1991). Este procedimiento en apariencia sencillo, aumenta su complejidad en forma proporcional al número de arcos y nodos del grafo, y ha logrado imponerse frente a otras metodologías y técnicas empleadas para el análisis de redes.

En principio se describen las principales característica del espacio seleccionado para su aplicación (Provincia de Corrientes), en el apartado metodológico se describen las medidas utilizadas que permiten conocer la estructura de la red (conectividad, accesibilidad y centralidad), luego se exponen los resultados de los cálculos de los índices (Beta, Gamma, Número Ciclomático, Alfa, Köning, Shimbel, G, Omega, Centralidad Media), las matrices (Conectividad, Accesibilidad), y la cartografía para lograr una descripción de las características topológicas de la red.

\subsection{Objetivos}

El presente trabajo se propone como objetivo general:

- Caracterizar la estructura de la actual red vial de la provincia de Corrientes mediante la aplicación de algunas medidas propias de la Teoría de Grafos.

Por otra parte se plantean como objetivos particulares:

- Analizar mediante el empleo de índices y matrices, la conectividad, accesibilidad y centralidad correspondiente al grafo de la red vial de Corrientes. 
- Realizar un aporte para la planificación, mejoramiento y aprovechamiento de la red vial.

\section{2. Área de estudio}

La Provincia de Corrientes se localiza en el noreste del país, entre los $27^{\circ}$ y $30^{\circ}$ latitud Sur y entre los $28^{\circ}$ y $30^{\circ}$ longitud Oeste. Se encuentra delimitada por dos importantes cursos fluviales: el río Paraná al Norte, la separa de la República del Paraguay, y al Oeste de las provincias de Chaco y Santa Fe; el río Uruguay al Este, que también sirve de límite internacional de nuestro país con las Repúblicas del Brasil y Uruguay, mientras que hacia el Sur, el río Guayquiraró lo separa de la Provincia de Entre Ríos(Figura No 1 ).

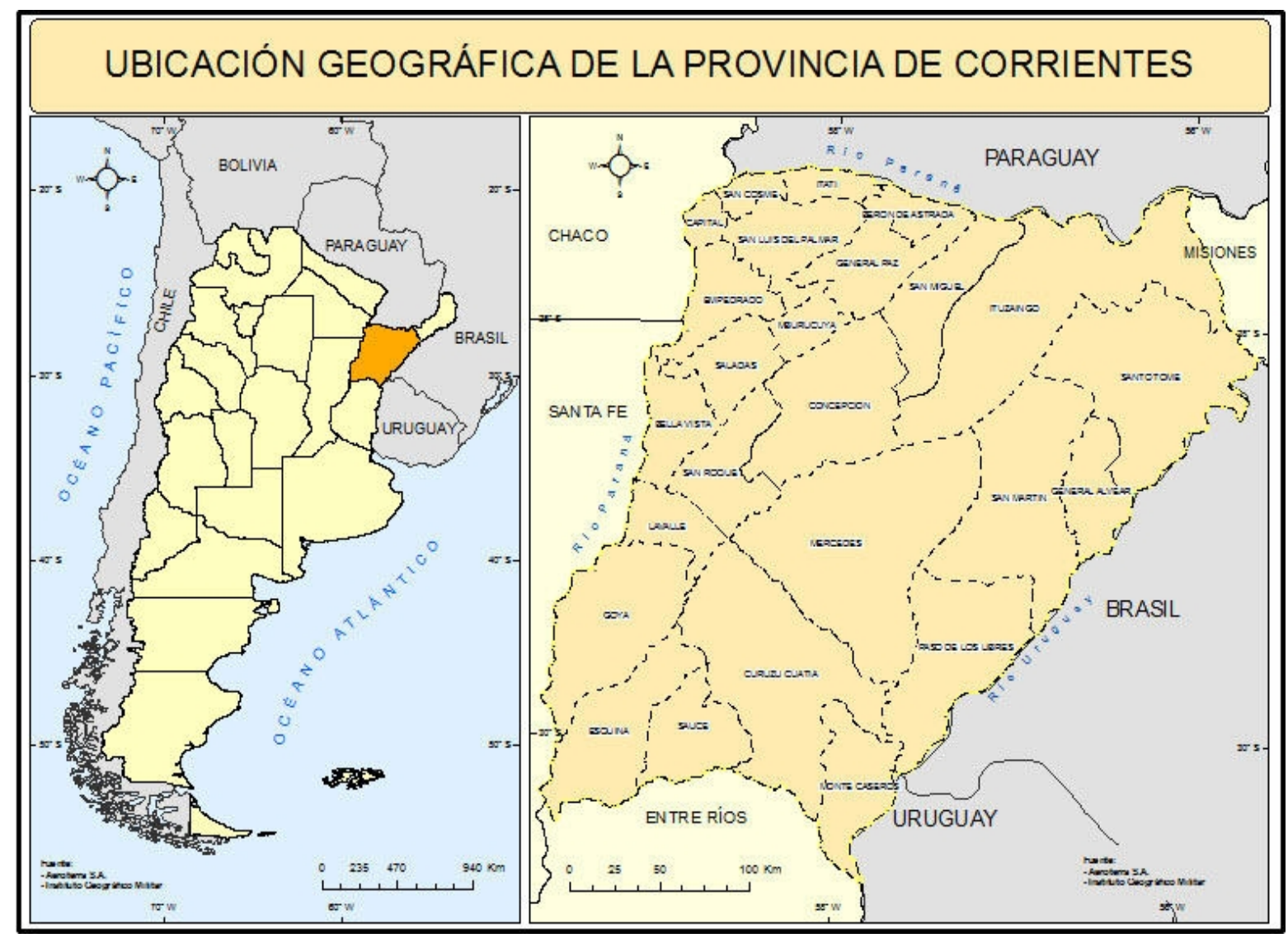

Figura 1. Ubicación geográfica de la Provincia de Corrientes en el contexto nacional. 


\section{MARCO CONCEPTUAL-METODOLÓGICO}

\subsection{Antecedentes}

Desde su introducción al campo de la Geografía por Garrison y Marble en los años ' 60 , se produjo una importante cantidad de material teórico-metodológico y aplicaciones concretas a muy diversas escalas y temáticas, aunque predominan claramente las referidas a redes de transporte. Referencias metodológicas obligadas sobre Teoría de Grafos en Geografía podemos mencionar las obras de Haggett y Chorley (1969), Tinkler (1977), Potrikovsky y Taylor (1984), Seguí Pons y Petrus Bey (1991), BosqueSendra (1992), Rodrigue (2006). En ellos se explica detenidamente la potencialidad (descriptiva o explicativa) de los diferentes tipos de índices que pueden aplicarse.

Fuera de la línea de análisis con grafos, pero en relación directa con el tema, escala y área de estudio, Pérez (1984) realiza una completa descripción de la evolución de la red vial provincial entre 1588 y 1898, por medio de una abundante cartografía que documenta la ocupación del espacio por la red de transporte, su relación con los centros poblados, y la influencia que supusieron los factores físico-naturales, humanos y económicos en su desarrollo.

En Geográfica № 12 (2004) se representan 8 planchas cartográficas con información referida al transporte en la Provincia, resultando de especial interés las referidas a la densidad de vías (accesibilidad) y la frecuencia del tránsito. La primera es calculada a partir de las longitudes de vías por unidad de superficie y donde se destacan varios focos (Noreste, Centro-Oeste, Sureste) de alta accesibilidad en el territorio provincial, así como un vacío en el área de los Esteros del Iberá; por otra parte, las de frecuencia del tránsito por las rutas (Tránsito Medio Diario y empresas por modos de transporte) fueron representadas en la cartografía por medio de líneas proporcionales, reflejando de forma clara el poder de atracción que ejerce la capital sobre las interacciones con el resto de las localidades.

Las experiencia de aplicación en el país se reducen a los trabajos de Natera Rivas (1995), analiza la red de vías de comunicación en las provincias del Nordeste Argentino (NOA), empleando para ello los índices Beta, Gamma, Número Ciclomático, Alfa, Número de Shimbel y el Índice Omega con el objeto de conocer las propiedades topológicas de los grafos. Es destacable el criterio empleadopara su construcción, considerando como nodos a las localidades de más de 2000 habitantes, a aquellas con menor cantidad (parajes o puestos), y por último los cruces de rutas pavimentadas sin población. Como conclusión se exponen las áreas de mayor y menor grado de accesibilidad del NOA, puntualizando el escaso desarrollo vial y las dificultades que se derivan.

Cardozo y otros (2009) realizan un análisis exploratorio-descriptivo de la red de transporte público en la ciudad de Resistencia por medio de una serie de índices propios de la Teoría de Grafos, y luego plasman en cartografía temáticapor medio de los Sistemas de Información Geográfica (SIG); de esta forma se logra una avance en 
el camino de la integración de las nuevas tecnologías geográficas y el desarrollo metodológico del análisis espacial con Grafos.

Un trabajo de aplicación realizado enAragón (España) por Garridos Palacios (1995), analiza la organización espacial de la red vial, mediante el empleo medidas de conexión, accesibilidad y centralidad. Este autor llega a la conclusión de que la red vial de la Comunidad Autónoma de Aragón presenta una alta conectividad, pero la misma se ve disminuida cuando el análisis se lo realiza a nivel provincial. La accesibilidad se presenta centralizada en el grafo y decrece a medida que se aleja del centro, representándola mediante curvas de isoaccesibilidad.

\subsection{Fuentes de Información y Criterios de Elaboración}

Para construir el grafo de la provincia de Corrientes se estableció una comparación entre los arcos y nodos con las rutas, las ciudades e intersecciones de rutas, por lo que el diseño resultante puede asemejarse al trazado real, pero a los fines de análisis topológicos no es necesario que así lo sea, la importancia radica en las relaciones o conexiones que se establecen.

Por otra parte, la escala de trabajo obligó a seleccionar las localidades con más de 2000 habitantes y las intersecciones de rutas, coincidiendo con el criterio empleado por Natera Rivas, para ello se utilizó el Censo Nacional de Población y Vivienda del año 2001. De ese modo se obtuvieron un total de 54 nodos.Las rutas también fueron sometidas a un proceso de selección, debido a su densidad, pavimentada y no pavimentada, y se ha optado por trabajarcon las primeras, tanto nacionales como provinciales, resultando un total de 64 arcos (Figura $\mathrm{N}^{\circ} 2$ ). El Mapa de la Red Vial de la Dirección de Vialidad de la Provincia de Corrientes y el Atlas de Rutas Firestonedel año 2008 sirvieron de fuentes de información para su elaboración. 


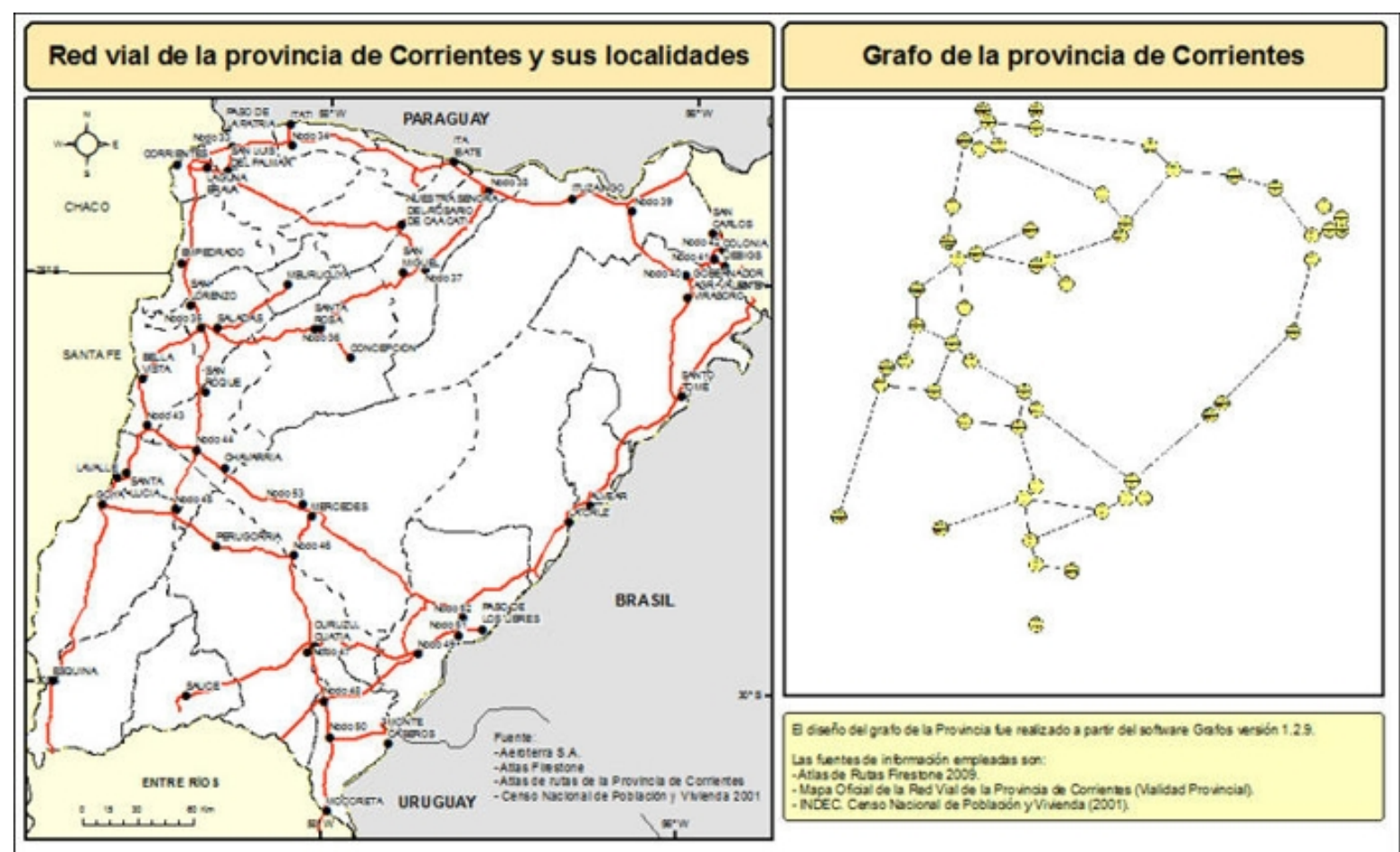

Figura 2. Red vial de la provincia de Corrientes con sus localidades y Grafo

Por otra parte, a causa de la falta de uniformidad en la terminología utilizada para designar a los elementos del grafo: nodo, vértice, nudo, para los elementos puntuales y arco, aristas, ejes, segmentos, para los elementos lineales, se ha decidido trabajar con arcos y nodos, guardando relación con la terminología empleada en los SIG.

\subsection{Medidas de Conectividad}

La conectividad o cohesión, determina el grado de comunicación recíproca entre los vértices, y es el grado de integración o interconexión que presenta una red para su funcionamiento interno. Parte del principio básico de que cuantos más arcos tenga el grafo, mayor será el grado de conectividad. En realidad, indica la mayor o menor complejidad estructural de la red, lo cual está ligado directamente al número de nodos, arcos y a su disposición espacial (Seguí Pons y Petrus Bey, 1991).

Entre los índices de conexión empleados figuran los siguientes:

- Índice Beta de Kansky

- Índice Gamma de Kansky

- Número Ciclomático

- Índice Alfa

El Índice Beta de Kanskyexpresala relación entre los arcos y nodos de un grafo. Donde (a) corresponde a los arcos y ( $n$ ) a los nodos. 


$$
\beta=\frac{a}{n}
$$

Este índice logra medir como aumenta la conectividad cuando se incrementa el número de arcosde un grafo(Seguí Pons y Petrus Bey, 1991). Las redes de transporte de estructura muy compleja asumen valores de índice $\beta$ más elevados, mientras las redes cuya estructura es más sencilla, tendrán valores más bajos(Potrykowsky, 1984).

El Índice Gamma relaciona el número de arcos existentes y el número máximo que puede existir dentro de un grafo determinado, teniendo en cuenta la cantidad de nodos de una red. Su fórmula se expresa de la siguiente manera:

$$
\gamma=\frac{a}{\frac{n *(n-1)}{2}}
$$

Donde (a) es el número de arcos y $(n)$ el número de nodos de una red.El valor resultante de este índice varía entre 0 para un grafo sin conexiones y 1 para un grafo completamente conectado.(Bosque Sendra, 1992).

Índice Gamma de Kansky(para los grafos planos): es otro índice de conexión que relaciona el número de arcos existentes y el mayor número de arcos posibles(Seguí Pons y Petrus Bey, 1991).Da una idea sobre las dimensiones de los complementos que se precisan incorporar a la red. Oscila entre 0 (ausencia de cohesión) y 1 (cohesión máxima)(Potrykowsky, 1984)su fórmula es la siguiente:

$$
\gamma=\left[\frac{l}{3} *(n-2)\right]
$$

El Número Ciclomático:determina la cantidad de circuitos que existen dentro de un grafo. Entendiendo por circuito, cada una de las múltiples maneras que existen para ir desde un nodo hasta el mismo, sin tener que pasar dos veces por el mismo arco. Se calcula restando el número total de arcos (a) al número de nodos necesario para formar un árbol (n-1), restándose además los grafos inconexos(g)que puedan existir (Bosque Sendra, 1992) su fórmula es la siguiente:

$$
\mu=a-(n-1)-g
$$

Para poder hacer comparable con otros grafos, al Número Ciclomático se debe hallar el Índice Alfa,el cual relaciona el número de circuitos observados en el grafo $(\mu)$ y los circuitos que existirán en caso de tratarse de un grafo completo.Su valor resultante varía entre 0 para un grafo sin ningún circuito y 1 para un grafo completo (Bosque Sendra, 1992)su fórmula es la siguiente:

$$
\alpha=\frac{\mu}{\left(2^{*} n-5\right)}
$$




\subsection{Medidas de Accesibilidad}

Las medidas de accesibilidad, permiten analizar la organización espacial de los nodos de un grafo, y los procesos de competencia que se establecen, jerarquizados según la facilidad de acceso desde cada uno en relación a los restantes nodos contenidos en el grafo (Garrido Palacios, 1995).

Dentro de las medidas de accesibilidad topológica podemos hallar:

- Matriz de Conectividad

- Matriz de Accesibilidad Topológica

- Número Asociado de Köning (NS)

- Número de Shimbel

- Índice G de Dispersión

El primer paso para determinar la accesibilidad de una red es la elaboración de un grafo, luego se procede a la construcción de la Matriz de Conectividad, una tabla simétricadonde se indica con 1 las relaciones directas entre nodos y con 0 la ausencia de una conexión directa. A partir de esta se obtiene la Matriz de Accesibilidad Topológica, en la cual se señalan la cantidad de arcos mínimos que son necesarios recorrer para ir desde un nodo a cada uno de los restantes (Seguí Pons y Petrus Bey, 1991).

El Número Asociado de Köningresulta serel valor mayor de cada fila en la Matriz de Accesibilidad, dicho número da una idea de la accesibilidad que posee el nodo de referencia con relación al nodo más lejano topológicamente. Cuanto menor sea el valor del Número Asociado, mayor será la accesibilidad del nodo (Garrido Palacios, 1995).

Además se puede definir elíndice deShimbel, que se obtiene sumando los valores de cada fila de la Matriz de Accesibilidad Topológica, lo que muestra la cantidad de arcos a atravesar para ir desde un nodo a todos los demás del grafo(Garrido Palacios, 1995)se expresa de la siguiente manera:

$$
A y=\sum d x y
$$

Donde $d x y e s$ el número de arcos (distancia) que separa los nodos $x$ e $y$ por el camino más corto.

A través del Índice de Shimbel es posible determinar otros dosíndices. El primero de ellos,denominado Longitud Media de laVíapor Potrykowsky (1984) y Garrido Palacios (1995), se trata de un cociente entre la accesibilidad topológica ( $A y)$ y el número total de nodos $(n)$, expresada de la siguiente manera:

$$
P y=\frac{A y}{n}
$$


Por otro lado, para evitar comparar redes con diferentes cantidades de nodos, se puede emplear el Índice de Accesibilidad Topológica Relativaoĺndice Omega(Garrido Palacios, 1995) definido como:

$$
\Omega_{g}=\frac{\left(A y-A_{\min }\right)}{A_{\max }-A_{\min } \overline{E !}} * 100
$$

Siendo $A y$ el Índice de Shimbel de dicho nodo, $A_{\min }$ el Índice de Shimbel de valor más bajo y $A_{\max }$ el de valor más alto. Los valores oscilan entre 0 y 100 para los nodos de mayor a menor accesibilidad, respectivamente (Garrido Palacios, 1995).

Para lograr una visión de conjunto del grafo en cuanto a la accesibilidad, se puede hallar el Índice $G$ de Dispersión, que se define como la suma de todos los Índices de Shimbel de cada nodo. Cuanto más alto sea el valor resultante, menor será la accesibilidad entre los nodos de la red.

Otro cálculo relacionado es la Accesibilidad Media de lared,que se obtiene dividiendo el Índice $\mathrm{G}$ de Dispersión por la cantidad de nodos $(n)$ del grafo (Bosque Sendra, 1992), y se presenta de la siguiente manera:

$$
A M=\frac{G}{n}
$$

\subsection{Medidas de Centralidad}

Las medidas de centralidad buscan reconocer la posición topológica de los nodos dentro del grafo para establecer su influencia sobre el área circundante.

La Centralidad Media de cada nodo es otro cálculo que permite reconocer la posición topológica de los nodos dentro de la red, así un nodo con valores mínimos demostrará ser central y, con altos valores poco central(Garrido Palacios, 1995). Su fórmula es la siguiente:

$$
C=\frac{A y}{(n-1)}
$$

\section{RESULTADOS}

A través de la elaboración del Grafo y las matrices, se han calculado diferentes índices que sirvieron para describir del estado general de la red vial de la provincia de Corrientes, atendiendo a los aspectos de conectividad, accesibilidad y centralidad. Una buena manera de presentar los resultados es a través de una cartografía temática que muestre las relaciones topológicas, de esa manera se identificaron lasáreas favorables y desfavorables. 


\subsection{Análisis de la conectividad, accesibilidad y centralidad}

Los índices Beta y Gamma de Kansky,Número Ciclomático e Índice Alfa son las medidas empleadas para analizar el estado de conectividad de la red.Debemos tener en cuenta que al grafo se lo considera en sus relaciones topológicas, por lo cual la realidad queda reducida al plasmarse en un dibujo. Por esto, el análisis es matemático y no establece relaciones directas con la realidad, sino que trata de definir el estado de la conectividad, accesibilidad y centralidad brindando una herramienta para la planificación de nuevas infraestructuras, como así también para su mejoramiento.

El índice Beta de Kanskyestablece una relación entre los arcos y los nodos que para el grafo de la provincia de Corrientes devuelve un valor de 1,19. Si el grafoincrementa la cantidad de arcos (rutas), también lo haría su valor, pues al ser un cociente y aumentarsolo el dividendo,la conectividad resultante se incrementa. Por ejemplo, si aumentamos las conexiones para igual número de nodos, observaremos el progresivo aumento del índice Beta $(\beta)$.

\begin{tabular}{|c|c|c|}
\hline $\begin{array}{c}\mathbf{N}^{\circ} \text { de } \\
\text { arcos }\end{array}$ & $\begin{array}{c}\mathbf{N}^{\circ} \text { de } \\
\text { nodos }\end{array}$ & $\boldsymbol{\beta}$ \\
\hline 10 & 54 & 0,18 \\
20 & 54 & 0,37 \\
40 & 54 & 0,74 \\
80 & 54 & 1,48 \\
\hline
\end{tabular}

El índice Gamma de Kansky nos da una idea del estado de cohesión que posee la red de la provincia de Corrientes, el valor devuelto muestra que la red se encuentra conectada en un $41 \%$, con lo cual es necesario incorporar un $59 \%$ más de complementos. Pero si bien topológicamente la red puede estar totalmente conectada, en la realidad esto puede parecer ineficiente debido al exceso de rutas. Este índice permite observar el aumento de lasconexiones, si se planificara una ruta nueva que facilite la comunicación entre áreas con baja y alta conectividad.

EINúmero Ciclomáticoindica la cantidad máxima de circuitos que se pueden hallar en el grafo. En nuestro caso el índice alcanza un valor de 11 circuitos.La importancia de este índice radica en que,cuantos más arcos se agreguen a la red más ciclos se sumaran y así mayor será la conectividad de la misma.

Ahora si queremos hacer comparable con otros grafos,se calcula el índice Alfa, valor que oscila entre 0 para una red sin circuitos a 1 para una red con todos los circuitos posibles. En el caso del grafo de la provincia de Corrientes el valor devuelto es de 0,214 con lo cual se puede llegar a decir que es bajo topológicamente. 
Por otra parte, las medidas de accesibilidad permiten analizar la disposición de los nodos y los arcos en el grafo por lo cual cada nodo se presenta jerarquizado según la accesibilidad que le corresponde.

Para determinar la accesibilidad, primero se construyó laMatriz de Conectividad(Tabla N¹). Luego se elaboró la Matriz de Accesibilidad Topológica, la cual considera el mínimo número de arcos que son necesarios para ir desde un nodo a los restantes. Así por ejemplo para ir desde la localidad de Paso de la Patria a Concepción es necesario atravesar 7 arcos, y para ir desde Paso de la Patria a Itatí3 arcos (Tabla N²).

Una vez construida la Matriz de accesibilidad Topológica se calculó el Número Asociado de Köningque resulta ser el valor más elevado de cada fila, ycuanto más elevado sea el valor, menor accesibilidad tendrá. Por ejemplo: las localidades de CaáCatí, Mocoretá y Monte Caseros son las que presentan los valores más altos (17), por lo que es menor la accesibilidad a las mismas. Al contrario, la localidad que menor valor presenta es Alvear con el máximo de accesibilidad (10).

Las variaciones en el índice de Shimbel oscilan entre losvalores de 524 en la localidad de San Carlos -con lo cual es el nodo menos accesible-y 301 para el nodo 44 (cruce de rutas Nacional º12 y Provincial N¹23), con la máxima accesibilidad.

El cálculo de laAccesibilidad Media devuelve un valor de 399, queindica el promedio de las distancias topológicas (números de arcos) que se debe recorrer en el grafo para desplazarse de un nodo cualquiera a todos los restantes (Figura $\mathrm{N}^{\circ} 3$ ). El otro índice calculadoes el de Accesibilidad Topológica Relativa o Índice Omega, que lo hace comparable con otros grafos. Este índice expresa en porcentaje la accesibilidad, siendo más accesible aquellos nodos que presenten menores porcentajes, como es el caso del nodo 44 con 0\%, seguido por la localidad de San Roque con un $3 \%$ al igual que el nodo № 35 (cruce de las rutas Nacional №12 y Provincial $\mathrm{N}^{0} 118$ ), y aquellos que presentan la menor accesibilidad con altos porcentajes, como las localidades de San Carlos con el 100\%,y Mocoretájunto a Monte Caseros con el 99\%.

Para lograr una visión de conjunto de la accesibilidad en la red, calculó el Índice $G$ de Dispersión, el cual resulta de la suma de los valores devueltos por el Índice de Shimbel de cada nodo. El valor resultante ha sido de 21.581 , equivalente al volumen total de conexiones mínimas presentes en el grafo.

Para poder analizar espacialmente los resultados se han elaborado representaciones cartográficas correspondientes a los índices obtenidos de la Matriz de Accesibilidad: Número Asociado de Köning, Índice de Shimbel, Índice Omega (isoaccesibilidad) eĺndice de Centralidad Media (isocentralidad).

El mapa de Número Asociado de Köning muestra que las localidades ubicadas en la margen Este de la Provincia, como Virasoro, Santo Tomé, Alvear, La Cruz, o los ubicados en el Centro, como Mercedes y Perugorría, o hacia el Noreste, Saladas, San Lorenzo y Empedrado presentan los valores más bajos, asociado a una mayor 
accesibilidad. Por el contrario, aquellos nodos que presentan mayores valores, tal es el caso de localidades como Esquina, Sauce, Mocoretá, Monte Caseros, San Carlos, Paso de la Patria e Itatípresentan una menor accesibilidad (Figura N³).

La Figura $\mathrm{N}^{0} 3$ muestra las localidades más accesibles según el Índice de Shimbel, como Corrientes, Empedrado, San Lorenzo, Saladas, Santa Rosa, San Roque, Bella Vista, Chavarría, Perugorría, Mercedes y las intersecciones de las rutas Nacional $\mathrm{N}^{0} 12$ y Provincial No123, Provinciales $\mathrm{N}^{0} 27$ y $\mathrm{N}^{0} 123$, Provinciales $\mathrm{N}^{0} 119$ y $\mathrm{N}^{0} 123$, Provinciales $\mathrm{N}^{0} 119$ y $\mathrm{N}^{0} 24$. Por otra parte en la periferia del grafo se localizan ciudades como Mocoretá, Monte Caseros, Esquina, San Carlos, Colonia Liebig's, etc., las cuales presentan una menor accesibilidad.

En el Mapa de Isoaccesibilidadseobservan áreas de elevados valores, tal es el caso de las localidades de San Carlos, Colonia Liebig's al Noreste, Monte Caseros,Mocoretáal Sureste, Esquina al Suroeste y Sauce al Sur, siendo éstas las menos accesibles. Por el contrario las localidades ubicadas en el Centro-Oeste, como San Roque, Chavarría y Mercedes presentanuna mayor accesibilidad (Figura No4). Por su parte la representación de la Isocentralidadmuestra las localidadesde Saladas, San Roque y Chavarría, y las intersecciones de las rutas Nacional No12 y Provincial $\mathrm{N}^{0} 123$ como lasde mayor centralidad, y por el contrario las localidades de San Carlos, Colonia Liebig's, Monte Caseros, Mocoretá son las que presentan la menor centralidad (Figura Nº4).

\section{CONCLUSIONES}

La Teoría de Grafos resulta una técnica adecuada cuando se pretende analizar las relaciones topológicas de una red (conexión, accesibilidad y centralidad), con lo cual la reducción de la realidad a un grafo (dibujo compuesto por arcos y nodos) facilita el análisis mediante el empleo de índices y matrices que ayudan a comprender el estado general de su estructura.

En lo que respecta a la conectividad, los índices calculados sobre la red vial de la provincia de Corrientes presentan niveles bajos, por lo que es posible determinar la magnitud de elementos que son necesarios implementar en la red para lograr el máximo de conectividad, aunque hay que tener en cuenta que una red con una alta conexión si bien es buena topológicamente, no será eficiente en la realidad. Los resultados arrojados permiten realizar comparaciones con otras redes e incluso analizar su evolución temporal o plantear mejoras para aumentar los niveles de conectividad.

En cuanto a la accesibilidad, la elaboración del mapa de isoaccesibilidad a partir del cálculo de los índices y matrices, permitió identificar áreas (Centro-Oeste) donde la accesibilidad es buena, como también áreas críticas, donde la accesibilidad disminuye localizadas preferentemente en sectores periféricos de la Provincia (Noreste, Sureste y Sur). 
Completan el análisis de la accesibilidad los mapas derivados del Número Asociado de Köning y el Índice de Shimbel. El primero de ellos ofrece una primera idea sobre la accesibilidad, ya que los valores devueltos señalanla proximidad topológica entre los nodos, pero mediante el segundo índice se logra una apreciación total de la accesibilidad de cada nodo.En sector CentroOeste de la provincia de Corrientes, se observan los nodos más accesibles.

Por último, el mapa de Isocentralidad Media delGrafo permite identificar aquellas localidades donde la centralidad es más alta,como Mercedes, Lavalle, San Roque, entre otras, ubicadas en el sector Centro Oeste provincial. La importancia de la centralidad es reconocer la facilidad de acceso que poseen estas localidades en la red.

Se evidencia que, desde el punto de vista topológico, la provincia de Corrientes manifiesta una gran accesibilidad y centralidad en el área Centro Oeste, disminuyendo hacia los sectores periféricos y por lo tanto dificultando su acceso. El vacío que constituyen los Esteros del Iberá se hacen notar en el grafo al no mantener una comunicación directa entre el Este y el Oeste de la Provincia. Resaltan en importancia las rutas pavimentadas nacionales ( $\mathrm{N}^{0} 12$ y $\left.\mathrm{N}^{0} 14\right)$ y provinciales ( $\mathrm{N}^{0} 123, \mathrm{~N}^{0} 118$ y $\mathrm{N}^{0} 119$ y otras menores como las $\mathrm{N}^{0} 5, \mathrm{~N}^{0} 6, \mathrm{~N}^{0} 24, \mathrm{~N}^{0} 27$, $\left.\mathrm{N}^{0} 120, \mathrm{~N}^{0} 126\right)$ que comunican las localidades de toda la provincia.

Este trabajo comprende un análisis de tipo descriptivo en su mayor parte, ya que caracteriza la red de la provincia de Corrientes, pero es también el punto de partida de los posteriores análisis que se deseen realizar, tales como de evolución multitemporal,la mejora u optimización de rutas existentes o proyectar nuevas vías de comunicación a la red, como la aplicación de índices más complejos procedentes de la Teoría de Grafos y la integración a los Sistemas de Información Geográficas (SIG).

\section{BIBLIOGRAFÍA:}

- Garrido Palacios, J. 1995.La Organización Espacial de la Red de Carretera en Aragón. Aplicación de la metodología de la Teoría de Grafos.Geographicalia.

Zaragoza. http://www.unizar.es/geografia/geographicalia/garridografos.pdf

- Natera Rivas, J. J. 1995.Acercamiento al Estudio de la Red de Vías de comunicación de las provincias del Nordeste Argentino. Geográfica, Instituto Panamericano de Geografía e Historia. 121:79:112.

- Potrykowsky, M. y Taylor, Z. 1984. Geografía del Transporte.Editorial Ariel, Barcelona.

- Seguí Pons, J. y Petrus Bey, J. 1991. Geografía de Redes y Sistemas de Transporte. Editorial Síntesis, Madrid.

- Bosque Sendra, J. 1992.Sistema de Información Geográfica. Rialp Editorial, Madrid.

- Pérez, M. E. 1984. La Red Vial y las Comunicaciones Terrestres en Corrientes. Origen y Evolución. 1588-1898. Cuadernos de Geohistoria 
Regional. Instituto de Investigaciones Geohistóricas. CONICETFUNDANORD, Resistencia. 10:169.

- Cardozo, O. Erica, G. y Parras, M. 2009. Teoría de Grafos y Sistemas de Información Geográfica Aplicados al Transporte Público de Pasajeros en Resistencia (Argentina). Revista Transporte y Territorio. Universidad de Buenos Aires. 1:89:111. Buenos Aires. www.rtt.filo.uba.ar/RTT00105089.pdf

- Haggett, P. y Chorley, R.J. 1969. Network Models in Geography. Edward Arnold, London.

- Rey, C. y Cardozo, O.(2007). Vulnerabilidad en Situaciones de Movilidad Urbana. Criterios Teóricos e Indicadores Válidos para su Estudio. En: Foschiatti (coord), Aportes Conceptuales y Empíricos de la Vulnerabilidad Global en el Nordeste Argentino. 320:334. Secretaría General de Extensión Universitaria. UNNE.Corrientes.

- Manoiloff, R. y Rey, W. (dir). 2004. Atlas Geográfico de la Provincia de Corrientes. Tomo V: Las Actividades Secundarias y Terciarias. 12:34 planchas. Instituto de Geografía. UNNE. 